\title{
Les cysticerques des animaux abattus à l'abattoir de Port-Bouet (Abidjan)
}

\author{
par G. S. MISHRA (*) et A. E. N'DEPO (**)
}

\begin{abstract}
RESUMÉ
Les auteurs exposent les résultats des observations faites à l'abattoir d'Abidjan sur les taux d'infestation des bovins, des ovins, des caprins et des porcins par C. bovis, C. tenuicollis et $C$. cellulosae. Ils étudient l'épidémiologie de ces affections, en soulignent l'importance économique et suggèrent la prophylaxie à mettre en cuvre pour en limiter l'étendue.

Ils font état de l'évolution de $C$. tenuicollis chez des chiots et des chatons expérimentalement infestés, qu'ils comparent avec les descriptions antérieurement faites par divers auteurs.
\end{abstract}

\section{MATÉRIEL ET MÉTHODES}

Chaque mois, depuis septembre 1976 à février 1978, 2 à 3 visites par mois sont faites à l'abattoir de Port-Bouet, A l'occasion de chaque visite, les cysticerques et autres parasites rencontrés chez les animaux abattus sont collectés, et apportés au laboratoire. Les kystes sont disséqués et les larves libérées des parois kystiques. Le scolex est aplati entre une lame et une lamelle. La fixation du scolex, ou des anneaux de cestodes aplatis, est faite au formol chaud à $5 \mathrm{p}$. 100. La coloration des spécimens est faite par la méthode classique: Carmin boracique, éclaircissement à l'essence de girofle, puis montage au Baume du Canada. Les coupes histologiques sont colorées à l'hématoxyline-éosine. Les cestodes adultes sont d'abord prélevés dans l'eau du robinet, lavés dans l'eau physiologique, puis tués par un bain de 10 minutes dans l'eau distillée chaude qui les tue en extension.

De jeunes chatons (Felis catis domesticus) et chiots (Canis familiaris) provenant de chattes

Service de Parasitologie du « Laboratoire de Pathologie Animale de Bingerville », B. P. 206 Bingerville, République de Côte-d'Ivoire.

(*) Expert FAO en parasitologie.

(**) Parasitologue national. et de chiennes errantes, sont élevés au laboratoire et nourris avec de la viande bouillie. Un examen coprologique des chiens et des chats, pratiqué systématiquement, a révélé la présence chez certains sujets, d'œufs d'Ascaris, d'Ankylostomes et de Coccidies classiques du genre Isospora.

L'infestation de chatons et de chiots des deux sexes, âgés de 1 à 4 mois, est réalisée par ingestion forcée de scolex frais, placés directement dans le pharynx, suivie par une gorgée d'eau pour faciliter le passage du scolex, sans être écrasé dans la bouche de l'animal. Les animaux sont sacrifiés de façon espacée dans le temps et les spécimens de cestodes obtenus sont systématiquement étudiés.

\section{RESULTATS}

\section{C. tenuicollis}

1184 sur 2420 moutons et 101 sur 185 chèvres sont porteurs de kystes, soit 49 p. 100 et 55 p. 100 d'infestation respectivement de ces petits ruminants par $C$. tenuicollis. Chez 49 p. 100 de sujets positifs, les kystes se trouvent dans la cavité abdominale, plantés dans le mésentère. A différentes reprises, les kystes ont été également trouvés dans le foie du même sujet (4 p. 100). 
Le nombre de kystes récoltés par animal est variable. Nous trouvons le plus souvent de 1 à 4 kystes par animal. Nous avons observé exceptionnellement quatre fois 30 kystes ; 5 fois 20 kystes et 1 fois 55 kystes, tous plantés dans le mésentère d'un animal.

Généralement, les kystes renferment deux parois : une externe qui est plus épaisse et de couleur crème-blanchâtre; l'autre plus mince, transparente et blanche.

A travers cette dernière, on voit un point blanc renfermant le scolex, invaginé dans le cou. L'intérieur du kyste est rempli d'un liquide clair et relativement fluide qui donne au kyste une consistance très molle et flexible. Sur une étude détaillée de 50 kystes, faite individuellement, nous avons noté une variation de taille entre 3 et $8 \mathrm{~cm}$ de circonférence (moyenne $5 \mathrm{~cm}$ ), poids entre 4 et $62 \mathrm{~g}$ (moyenne $21 \mathrm{~g}$ ) et le volume de liquide entre 4 et $42 \mathrm{ml}$ (moyenne $18 \mathrm{ml}$ ). Le liquide a une densité de $1007 \mathrm{~g} / \mathrm{cm}^{3}$ à une température de $23^{\circ} \mathrm{C}$ déterminée par le picnomètre, et un $\mathrm{pH}$ presque neutre $(6,8)$. La larve, libérée de ses enveloppes présente un scolex muni de 30 à 34 crochets (petits et grands) et quatre ventouses de diamètre $0,30-0,32 \mathrm{~mm}$. Les grands et les petits crochets mesurent de 0,15 à 0,2 et 0,11 à $0,12 \mathrm{~mm}$, respectivement.

\section{$1.1 T$. hydatigena}

Il nous a été possible d'observer la migration et le développement de Taenia hydatigena et de le récolter dans le tube digestif des deux espèces animales suivantes:

a) Chez le chien : 9 chiots ont été autopsiés en l'espace de $7,12,15,30,45,60,95,110$ et 126 jours après infestation. Le chien $n^{\circ} 1$ est mort le $7^{e}$ jour avec une forte diarrhée liquide, déshydratation et amaigrissement. Le $7^{\mathfrak{e}}$ animal a été observé, éliminant la première chaîne de ténia le $80^{\mathrm{e}}$ jour d'infestation. Chez les animaux autopsiés, le nombre de $T$. hydatigena obtenu par rapport au nombre de kystes ingérés nous donne un taux moyen d'infestation de 85 p. 100 . Le chien est réceptif à cette infestation à raison de 100 p. 100 .

Nous n'avons pas observé une différence significative entre la taille du rostre, des ventouses, ainsi que la taille et le nombre des grands et petits crochets récoltés chez les moutons et ceux de $T$. hydatigena développés chez les chiens. Nous avons noté une croissance de taille du ver entier de $3 \mathrm{~mm} \mathrm{le} 12^{\circ}$ jour à $1470 \mathrm{~mm}$ le $207^{\mathrm{e}}$ jour d'infestation.

La formation des cufs nous semble très tardive. L'essentiel du développement génital se fait donc en grande partie entre le $30^{\mathrm{e}}$ et le $60^{\mathrm{e}}$ jour d'infestation et plus tard. Le $80^{\mathrm{e}}$ jour, nous observons l'élimination spontanée d'anneaux gravides dans les excréments du chien.

Les jeunes chiens infestés présentent dès le $5^{\mathrm{e}}$ jour après le repas infestant, un ralentissement de la croissance pondérale à raison de $7 \mathrm{~g}$ en moyenne par jour par rapport au témoin. Puis l'animal a un gain de poids normal à raison de $18 \mathrm{~g}$ en moyenne par jour dès que son état général s'est rétabli vers le $30^{\mathrm{e}}$ jour. Au début de l'infestation, l'état général est altéré, le poil est piqué et terne. L'animal préfère rester isolé et manifeste une anorexie. On observe de façon épisodique des périodes de constipation et de diarrhée, quelquefois profuse. En ce qui concerne l'intensité des symptômes observés, la situation s'avère critique au début de l'infestation. Le tableau clinique s'est aggravé une seule fois, le sujet est mort au 7 e jour refusant toute nourriture et manifestant une diarrhée liquide et une déshydratation aiguë. A l'autopsie, nous n'avons pas trouvé de ténia. Par contre, 13 spécimens de Toxocara canis ont été isolés chez ce sujet. Enfin, ce cas paraît extrême, mais en général, l'infestation par T. hydatigena, à la dose expérimentale, est relativement bien supportée par les chiots hormis des troubles digestifs passagers.

b) Chez le chat: 20 jeunes chats ont été infestés par des kystes variant au nombre de 15 à 55 et ont été autopsiés à des périodes d'infestation différentes. Des ténias de $1,4,5,9$, 10,11 et 38 jours ont été isolés dans l'intestin grêle de $7 / 20$ sujets. Ce rapport représente une susceptibilité des chats à l'infestation de $T$. hydatigena au niveau de $35 \mathrm{p} .100$. Chez ces animaux positifs, le nombre de $T$. hydatigena obtenu en rapport du nombre de kystes ingérés, nous donne un taux moyen d'infestation de 53 p. 100.

Dès le premier jour d'infestation, nous constatons quelques modifications morphologiques du parasite qui en font un ver actif, pouvant se fixer dans la paroi de l'intestin, les crochets et les ventouses saillants, le scolex fonctionnel et le cou se différenciant. La taille moyenne du ver oscille entre $1,6 \mathrm{~mm}$, le premier jour et $55 \mathrm{~mm}$ le $38^{\circ}$ jour. Le rostre, les ventouses et les crochets ne présentent pas une différence de taille importante. Par contre, les anneaux ne 
subissent pas un accroissement classique : ils commencent à se lyser même à partir du $23^{\circ}$ jour. Une seule fois (chat $n^{\circ} 18$ ) nous avons trouvé 4 cestodes sur 20 kystes présentant des anneaux en voie de développement. Nous n'avons pas trouvé de cestodes au-delà du $39^{\circ}$ jour dans l'intestin des chats observés jusqu'au $110^{\mathrm{e}}$ jour d'infestation.

Les chatons infestés présentent dès le premier jour après le repas infestant, les symptômes comme les jeunes chiens, une chute de croissance de poids ; le poil piqué et terne, une anorexie, l'animal est triste, il reste isolé. On observe de façon épisodique des périodes de vomissements, de diarrhée parfois profuse, d'anémie, de lacrimation et d'amaigrissement.

Si l'animal survit à la crise pendant ces 10 premiers jours d'infestation, il reprend son état normal, tout rentre dans l'ordre et on constate une croissance de poids à raison de $5,5 \mathrm{~g}$ par jour.

\section{C. cellulosae}

Durant la période de notre enquête, 99 porcs sur 3924 ont été reconnus porteurs de vésicules ladres, soit 2,5 p. 100. Dans la majorité des cas, la cysticercose est généralisée et particulièrement intensifiée dans la région scapulaire et la cuisse arrière.

Le détail de nos observations sur la dispersion épidémiologique de la cysticercose porcine est résumé dans le tableau $\mathrm{I}$.
Morphologiquement, le cysticerque est solide et il ressemble à une petite bulle enveloppée dans une membrane blanche et transparente qui comporte quelques gouttes de liquide clair. La masse kystique elle-même a une dimension moyenne de $4 \mathrm{~mm}$ et avec sa couverture qui lui donne une forme allongée, elle mesure $8,2 / 4 \mathrm{~mm}$. Une vingtaine de cysticerques ont été disséqués et les crochets et ventouses comptés et mesurés. Le nombre de crochets oscille entre 26 et 28 par rostre, les grands crochets mesurent, en moyenne $0,15 \mathrm{~mm}$ et les petits crochets $0,11 \mathrm{~mm}$. Les ventouses ont une dimension de $0,25 \mathrm{~mm}$.

\section{C. bovis}

Les grands ruminants abattus sont en majorité de race Zébu, fréquemment N'Dama et rarement Baoulé.

Les zébus proviennent des pays voisins. Ils traversent la Côte-d'Ivoire pour arriver à Abidjan, tandis que les N'Dama et Baoulé sont des animaux la plupart élevés en Côte-d'Ivoire.

De 1976 à 1978, l'examen de 10270 bovins adultes, de race, sexe et provenance différents a été effectué, parmi lesquels 90 animaux ont été reconnus porteurs de Cysticercus bovis, soit 0,8 p. 100 d'infestation naturelle. Très souvent, les kystes ont été isolés dans le ccur (myocarde) et la langue. Ces organes sont saisis. A 15 reprises, nous avons rencontré les cysticerques dans le foie. La cysticercose généralisée

TABL. $\mathrm{N}^{\circ}$ I-Infestation naturelle des porcs par Cysticercus celiuiosae.

\begin{tabular}{|c|c|c|c|}
\hline $\mathrm{R}$ è $\mathrm{g}$ i o n & $\begin{array}{c}\text { Nombre de porcs } \\
\text { examinés }\end{array}$ & $\begin{array}{l}\text { Nombre de porcs } \\
\text { porteurs de kystes }\end{array}$ & $\begin{array}{c}\text { Pourcentage } \\
\text { d'infestation }\end{array}$ \\
\hline Abidjan & 394 & 1 & \\
\hline Adzope & 14 & 0 & \\
\hline Anyama & 11 & 0 & \\
\hline Bingerville & 465 & 0 & \\
\hline Bimbregso & 56 & 0 & \\
\hline Bouaké & 507 & 29 & \\
\hline Daloa & 18 & 0 & \\
\hline Grand-Bassam & 613 & 1 & \\
\hline Jacqueville & 25 & 0 & \\
\hline Korhogo & 1763 & 68 & \\
\hline Port-Bouet & 58 & 0 & \\
\hline Tota 1 & 3924 & 99 & 2,5 \\
\hline
\end{tabular}


est rare et nous en avons détecté seulement dans 21 cas.

Les kystes sont, généralement de forme ovoïde, à paroi mince, translucide, renfermant quelques gouttes (1-3) de liquide d'une couleur rougeâtre. A travers la paroi kystique, on note une tache punctiforme blanchâtre, opaque qui correspond à l'invagination céphalique. Les kystes intacts mesurent de 5-12 × 4-8 mm (moyenne $11,3 / 7 \mathrm{~mm}$ ). Après dissection et aplatissement du scolex entre lame et lamelle, nous avons noté la présence d'un scolex d'environ $1 \mathrm{~mm}$ de diamètre, de 4 ventouses de 0,25 à $0,3 \mathrm{~mm}$ de diamètre et l'absence complète d'un rostre et de crochets.

\section{Kystes hydatique ou échinocoque (Echinococcus polymorphus)}

Sur 1520 animaux, nous avons isolé à 36 reprises dans les reins, soit 2,3 p. 100 d'infestation et à 2 reprises dans le foie $(0,13$ p. 100$)$ de bovins. Les kystes ont une taille de 5 à $10 \mathrm{~cm}$ de long et sont lobulés. A la dissection, tous les kystes ont été trouvés stériles et aucun ne présentait les scolex des cestodes.

\section{DISCUSSION ET CONCLUSIONS}

A notre connaissance et depuis 1956, aucune publication n'a été effectuée sur la cysticercose des petits ruminants en Afrique.

Nous pensons, dans ce premier rapport, que les taux de 40 p. 100 et 55 p. 100 de cysticercose ovine et caprine respectivement, sont importants et que leur incidence doit être, sans nul doute, une affaire de conséquence sur l'importante population canine domestique ou sauvage, qui joue un rôle dans la dissémination de cette maladie en Côte-d'Ivoire et dans ses voisinages.

Sur le plan général de la pathologie, les cysticerques détruisent les organes pendant leur migration avant de s'installer dans un organe ou dans la cavité abdomino-thoracique. De ce fait, ils provoquent la pneumonie cysticercose, l'hépatite cysticercose comme les douves, l'atrophie du foie, la péritonite, l'ascite, l'urémie et éventuellement la mort de l'animal, la plupart du temps sans aucun signe clinique spécifique. C'est donc une maladie qui n'est pas décelable du vivant de l'animal. Cependant, il est indispensable de prendre des mesures sanitaires strictes dans les élevages de petits ruminants, et d'éviter l'approche des carnivores sauvages ou domestiques sans les avoir déparasités.

Chez tous les chiots infestés expérimentalement par des kystes, se sont développés des ténias à raison de 80 à 100 p. 100 . Par contre, les chatons ne démontrent une susceptibilité à cette infestation qu'à 35 p. 100 seulement. D'une façon globale, le nombre et la taille des scolex, rostre, ventouses et crochets, ne varient pas chez ces deux hôtes et demeurent tels qu'ils se trouvent dans l'état kystique (larvaire). Le nombre des crochets est de 30 à $34 \mathrm{chez}$ les kystes ou chez les ténias adultes, qui correspondent au rapport de PANEBIANCO (8) et SOULSBY (9). La croissance des segments de ténia subit un développement classique et progressif dans le tube digestif de son hôte définitif (chien), tandis qu'ils ne se développent pas d'une façon normale dans le tube digestif du chat, très probablement parce que ce dernier est un hôte anormal ou accidentel ou temporaire pour ce ténia.

Dans une étude expérimentale comparable à la note sur le développement de $T$. hydatigena chez le chien, FEATHERSTON (3) pendant ses études sur 90 jours a observé le pourcentage de développement de ce ver chez son hôte normal à raison de 80 p. 100 , une strobilation le $7^{\mathrm{e}}$ jour, l'apparence des testicules le $20^{\mathrm{e}}$ jour, la fertilisation des œufs le $25^{\circ}$ jour, la maturation des œufs le $48^{\circ}$ jour et l'expulsion des segments le $56^{\mathrm{e}}$ jour après l'infestation. Nos chiens expérimentaux ont été également susceptibles à cette infestation à raison de $80-100$ p. 100 , la formation des organes génitaux a été observée entre le $30^{\mathrm{e}}$ et le $60^{\mathrm{e}}$ jour, mais l'expulsion volontaire des premières chaînes d'anneaux ne s'effectuait que le $80^{\mathrm{e}}$ jour après le repas infestant.

En ce qui concerne l'infestation des chats par ce cestode, nous citerons les travaux des deux auteurs, notamment celui de BULJEVIC (2) qui a infesté un chat de 3 mois d'âge, par 3 repas infestants succincts, composés de 2 cysticerques provenant du foie d'un porc, puis 2 cysticerques trouvés chez le mouton et, le $7^{e}$ jour par 4 kystes isolés dans le foie d'un porc. L'auteur a trouvé le premier anneau de ténia dans un excrément de chat le $67^{\circ}$ jour après le dernier jour d'infestation et à l'autopsie le $71^{\mathrm{e}}$ jour 2 adultes de $T$. hydatigena mesurant $68-76 \mathrm{~cm}$ dans l'intestin grêle de ce chat. L'auteur conclut que le chat est un hôte favorable pour ce cestode. D'autre part, SWEATMAN et collab. (10) constatent 
que le chien est un seul carnivore porteur de $T$. hydatigena et que le chat pourrait s'infester par ce parasite, mais celui-ci n'atteint pas sa maturité chez cet animal. Nos observations sont en accord avec celles de ces derniers auteurs. SOULSBY (9) signale que le chat est un hôte probable de ce ténia. Si ce cestode peut atteindre son état adulte, il est à considérer que le chat peut jouer un rôle dans la dissémination de ce parasite, nous n'avons pu le trouver durant nos études.

L'importance de la cysticercose porcine et bovine est indiscutable au point de vue zoonose (cestodose humaine d'origine animale). L'échange de ce cestode entre l'homme et l'animal et la contamination de ces deux vertébrés est une question purement sanitaire. En ce qui concerne l'infestation du porc par $C$. cellulosae et du bovin par $C$. bovis, c'est l'homme qui en est essentiellement la source. Par contre, le tenia humain à $T$. solium est à l'origine du porc, du sanglier et du chien (dans la population où la cynophagie est en pratique) et le taenia humain à $T$. saginata est toujours d'origine bovine et rarement dans la consommation des mammifères : antilopes, gazelles, girafes, etc... dont la viande n'est pas soumise à l'inspection sanitaire.

Les rapports sur l'étude épidémiologique similaire de la ladrerie bovine due à $C$. bovis en Afrique sont donnés par les auteurs notamment ; GRABER (4) Afrique du Sud 2,8 p. 100, Cameroun $15-20$ p. 100 , Ethiopie 80 p. 100 , Guinée 20 p. 100, Ituri (Zaïre) 70 p. 100, Kenya
$30-36$ p. 100 , Madagascar $4-20$ p. 100 , Oubangui 30-68 p. 100, République Voltaïque 7 p. 100 , Ruanda 15 p. 100, Sénégal 0,3-2 p. 100, Sierra Leone 38 p. 100 , Soudan 0,8 p. 100, Tchad 15,5 p. 100 ; MITCHELL (7) Ugadam (Karamoja) 29 p. 100 ; WALKER (11) Matsapa (Swaziland) 18 p. 100 et HEEVER et collab. (6) Afrique du Sud 3,6 p. 100. En ce qui concerne la ladrerie porcine due à $C$. cellulosae en Afrique, nous citons les travaux de GRABER et collab. (5) au Tchad 7 p. 100 et BOWLES et collab. (1) en Afrique du Sud qui ne mentionnent pas le taux d'infestation.

La notion préliminaire sur l'incidence de la ladrerie porcine $(2,5 \mathrm{p}$. 100) et la ladrerie bovine ( 0,8 p. 100) détectée chez les porcs et les bœufs abattus à l'abattoir de Port-Bouet, Abidjan (Côte-d'Ivoire) nous semblent atteindre un taux d'infestation important dans la dissémination des ténias humains qui reflètent l'existence de cette zoonose dans cette région d'Afrique.

\section{REMERCIEMENTS}

Nous remercions le personnel du service de parasitologie de Bingerville pour son aide constante, les Directeurs du Laboratoire (Dr. J. CHAMBRON et Dr A. ANGBA) pour les facilités apportées ainsi que les Directeurs (Dr. F. BAMBA et Dr B. TAURE) et le personnel de l'abattoir de Port-Bouet pour leur aide pendant toute la durée de notre enquête.

\section{SUMMARY}

\section{Cysts of animals slaughtered at Port Bouet Abattoir (Abidjan)}

An epidemiological survey on the cysticercosis of food animals slaughtered at Port-Bouet Abattoir (Abidjan) was carried out during 1976-78. The cysts recovered and the percentage of animals infested with such cysts were as follows : $C$. tenuicollis : sheep 49 p. 100 , goat 55 p. $100 ; C$. cellulosae : pig 2,5 p. $100 ; C$. bovis : cattle 0,8 p. 100 ; Hydatid cyst : bovine kidney 2,3 p. 100 bovine liver 0,13 p. 100 .

In 9 young pups and 20 kittens experimentally infected with $C$. tenuicollis, it was observed that : 80 to 100 p. 100 of cysts develop normally into $T$. hydatigena in every dog infected which prooved to be a good definitive host. The gravid segments were seen in the faeces of a dog on 1ts 80th day of infection. 35 p. 100 of cats are susceptible to this infection where $T$. hydarigena does not develop fully as such this carnivore may be accepted as an accidental, occasional or temporary host for this taenia.

\section{RESUMEN}

Los cisticercos de los animales matados en el matadero de Port-Bouet (Abidjan)

Una encuesta sobre la presencia de los cisticercos en los animales matados en el matadero de Port Bouet de Abidjan (Costa de Marfil), efectuada de 1976 
a 1978 , demuestra una tasa de infestación por $C$. tenuicollis : de 49 p. 100 en las ovejas, 55 p. 100 en las cabras ; por C. cellulosae : 2,5 p. 100 en los cerdos : por $C$. bovis : de 0,8 p. 100 en los bovinos; quistes hidáticos : 2,3 p. 100 en los tiñones y 0,13 p. 100 en los higados de bovinos examinados.

El estudio sobre la evolución de $C$. tenucollis en 9 cachorros y 20 gatitos experimentalmente infestados en el laboratorio demuestra que el perro, con una receptividad de 80 a 100 p. 100 , es un huésped definitivo en el cual Cysticercus tenuicollis se desarrolla y se vuelve tenia hydatigena y llega a su madurez normal. Se encuentró la prımera cadena grávida de dicho tenia en el excremento de un perro el $80^{\circ}$ día después de su infestación. El gato, en el cual el tenia se desarrolla muy 1rregularmente, tiene una susceptibilidad de 35 p. 100 a dicha infestación. No se encuentró jamás el estado adulto de este céstodo en dicho animal que podría ser un huesped accidental, occasional o temporario para este céstodo.

\section{BIBLIOGRAPHIE}

1. BOWLES (J.), VESTER (A.), PIENAAR (J. G.), HEEVER (L. W.) VAN DEN. Visceral localization of C. cellulosae. J. S. Afr. Vet. Ass., 1972, $43(3)$ : 299-300.

2. BULJEVIC (S.). Ulogo naveke u epizootioloji T. hydatigena (Pallas 1877). Veterinarski Glasnik Belgrade, 1960, 14 (9) : 677-678.

3. FEATHERSTON (D. W.). T. hydatigena 1. Growth and development of adult stage in the dog. Expl. Parasit., 1969, 25 : 329-338.

4. GRABER (M.). La cysticercose bovine. Son importance dans les zones sahéliennes d'élevage de la République du Tchad. Rev. Elev. Méd. vét. Pays trop., 1959, 12 (2) : 121-148.

5. GRABER (M.), CHAILLOUX (A.). Existence au Tchad de la ladrerie porcine à $C$. cellulosae (Rudolphi). Rev. Elev. Méd. vét. Pays trop., 1970, 23 (1) : 49-55.

6. HEEVER (L. W.) VANDEN. The degree of cysticer- cosis infestation of cattle in terms of standard meat inspection procedures. $J . S$. Afr. vet. med. Ass., 1969, 40 (1) : 47-49.

7. MITCHELL (J. R.). Some aspects of epidemiology and prevalence of $C$. bovis in Africa. Bull. epiz, Dis. Afr., 1973, 21 : 133-143.

8. PANEBIANCO (F.). Ricerche e considerazioni sul numero degli uncini del C. tenuicollis. Alli Soc. Ital. Sci. Vet., 1950, $4: 471-476$.

9. SOULSBY (E. J. L.). Helminths, Arthropods and Protozoa of Domestic Animals, VI ed., London, Baillère, Tindall and Cassel, 1971, $120 \mathrm{p}$.

10. SWEATMAN (G. K.), WILLIAMS (R. J.). Wild animals in New Zeeland as hosts of Echinococcus granulosus and other taeniid tapeworms. Trans. r. Soc. New Zeeland. Zoology, 1962, 2 (26) : 221-250.

11. WALKER (W. D.). Some observations on $C$. bovis at Matsapa abattoir in Swaziland. J. S. Afr. Vet. Ass., $1972,43(2)$ : 197-199. 